\title{
Patterning effects in quantum dot amplifiers characterized through pump probe spectroscopy using two pump pulses
}

Berg, Tommy Winther; Poel, Mike van der; Laemmlin, M.; Bimberg, D.; Birkedal, Dan

Published in:

2004 IEEE LEOS Annual Meeting, Conference Proceedings

Link to article, DOI:

10.1109/LEOS.2004.1363415

Publication date:

2004

Document Version

Publisher's PDF, also known as Version of record

Link back to DTU Orbit

Citation (APA):

Berg, T. W., Poel, M. V. D., Laemmlin, M., Bimberg, D., \& Birkedal, D. (2004). Patterning effects in quantum dot amplifiers characterized through pump probe spectroscopy using two pump pulses. In 2004 IEEE LEOS Annual Meeting, Conference Proceedings (pp. 667-668). IEEE. https://doi.org/10.1109/LEOS.2004.1363415

\section{General rights}

Copyright and moral rights for the publications made accessible in the public portal are retained by the authors and/or other copyright owners and it is a condition of accessing publications that users recognise and abide by the legal requirements associated with these rights.

- Users may download and print one copy of any publication from the public portal for the purpose of private study or research.

- You may not further distribute the material or use it for any profit-making activity or commercial gain

- You may freely distribute the URL identifying the publication in the public portal 


\title{
Patterning effects in quantum dot amplifiers characterized through pump probe spectroscopy using two pump pulses
}

\author{
T. W. Berg ${ }^{1}$, M. van der Poel ${ }^{1}$, M. Laemmlin ${ }^{2}$, D. Bimberg ${ }^{2}$, and D. Birkedal ${ }^{1}$ \\ ${ }^{1}$ Research Center COM, Technical University of Denmark, Build. 345V, DK-2800 Kgs. Lyngby, Denmark \\ Phone: +45 4525 6379, Fax: +454593 6581, E-mail: twb@com.dtu.dk \\ ${ }^{2}$ Institut für Festkörperphysik, Technische Universität Berlin, Germany
}

Abstract: Patterning effects at ultrahigh bitrates in quantum dot amplifiers are investigated by a novel experimental technique. Slow carrier dynamics in the excited states and wetting layer are found to limit the potential for ultrahigh-speed signal processing.

Semiconductor optical amplifiers (SOAs) with quantum dots (QDs) as the active medium have been shown to possess great potential as both linear amplifiers with high saturation power and broad gain spectrum $[1,2]$ and as nonlinear elements to be used for optical signal processing [3]. The experimental demonstration of ultrafast and complete gain recovery on a timescale of less than a picosecond a few years back [4] indicated the potential for ultrahigh-speed signal processing in these devices. However, as pointed out theoretically [5], the depletion of the carrier reservoir in the wetting layer (WL) and barrier regions following the amplification of a pulse means that despite the fast initial gain recovery a strong pattern effect might be introduced when the amplifier responds to a train of pulses. It is essential to understand the interaction between neighboring pulses at short time delays in order to understand the fundamental limitations to ultrahigh-speed signal processing in these devices.

The present work investigates experimentally the interaction between two pulses delayed by a variable time with respect to each other. This is done through pumpprobe spectroscopy, which allows for a high time resolution and therefore gives an accurate picture of the gain dynamics during the amplification process. The setup used is described in detail in [6] with the exception that the pump pulse is here split in two by a cube beamsplitter and one of the resulting pump pulses is delayed through a variable delay stage. Since the delay between the two pump pulses can be reduced to any value desired, it is by this

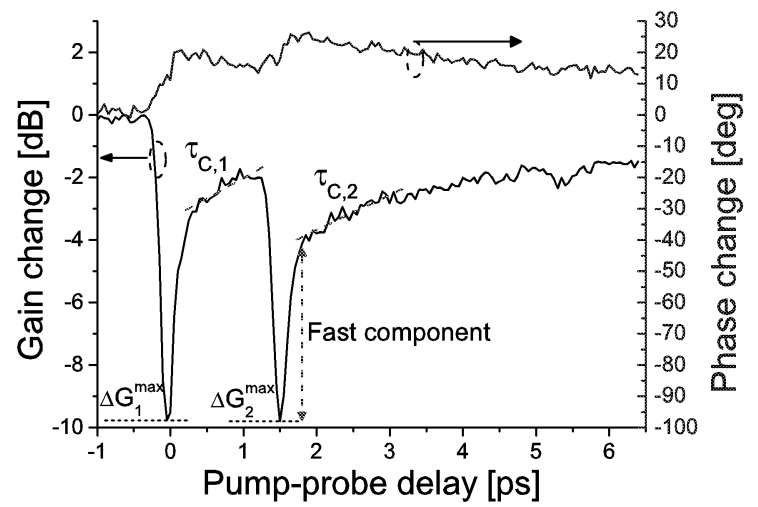

Fig. 1. Gain and phase changes during amplification of two pump pulses separated by $1.5 \mathrm{ps}$ with respect to each other at a bias current of $60 \mathrm{~mA}$. method possible to investigate the mechanisms behind patterning effects even for bit rates in the Tbit/s regime. The fundamental limit is determined by the width of the pump pulses, which is on the order of $130 \mathrm{fs}$.

The device used for this investigation is a ridge waveguide (width $7 \mu \mathrm{m}$, length $2 \mathrm{~mm}$ ) SOA with 5 sheets of self-organized, MBE grown InAs/GaAs QDs as active material [7]. The end facets of the amplifier are angled to suppress optical feedback.

Fig. 1 shows the resulting gain and phase changes experienced by the probe in response to the presence of the two pump pulses, which are separated by $1.5 \mathrm{ps}$, at the wavelength $1250 \mathrm{~nm}$, close to the QD peak emission wavelength. Both pump pulses induces a maximum gain change at the peak, $\Delta G_{1}^{\max }$ and $\Delta G_{2}^{\max }$ slightly above -10 $\mathrm{dB}$. At the input pulse energies used in this case a significant part of the instantaneous gain compression is caused by two-photon absorption. The gain recovery after each pulse consists of two distinctly different regimes. A fast gain recovery on a timescale of $150 \mathrm{fs}$, which is attributed to two-photon absorption and fast intra-dot carrier relaxation, followed by a slower component attributed to capture of carriers from the WL into the dots. We denote the timescale of recovery immediately following the fast component as the capture times, $\tau_{C, 1}$ and $\tau_{C, 2}$.

It is clear that the presence of the first pump pulse

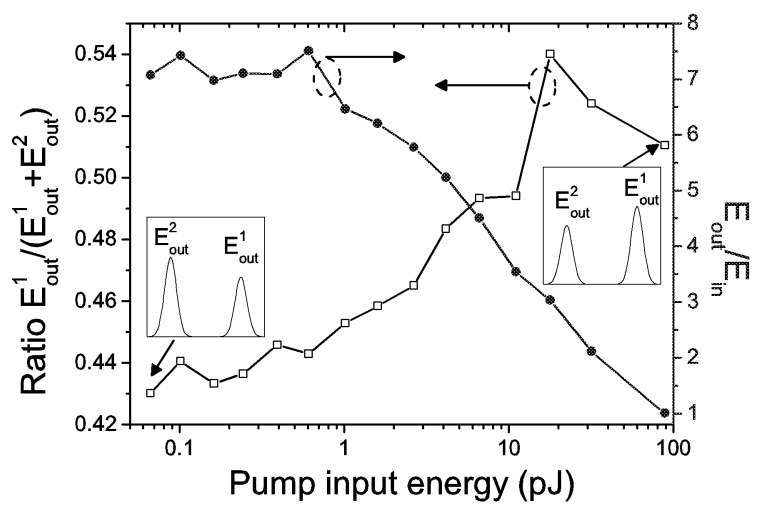

Fig. 2. Pulse energy of first pump pulse relative to the total energy of first and second pump pulse (left axis) and on-chip single pulse gain (right axis) at a current of $42 \mathrm{~mA}$. Input energies are measured before coupling into the device, i.e. no coupling is accounted for. 
influnces both the gain and refractive index seen by the second pump pulse due to slow recovery component at this combination of input power and bias current. This influence is reflected in the ratio of the output pulse energies of the two pump pulses. The ratio of the output energy of the first pump pulse, $E_{\text {out }}^{1}$ to the sum of the two pulse energies $E_{\text {out }}^{1}+E_{\text {out }}^{2}$ is shown in fig. 2 (left axis) as function of input pulse energy. In the small-signal regime the ratio reaches a nearly constant value around 0.43 indicating smaller output energy for the first pulse compared with the second pulse. The difference in this regime is due to slightly different coupling efficiencies to the waveguide for the two pulses. As the input power is increased, the ratio starts to increase due to gain saturation, i.e. the first pulse saturates the gain causing an increasingly smaller amplification of the second pulse. The ratio of the output pulse energy of a single pulse relative to the corresponding input energy, proportional to the on-chip single pulse gain, is shown on the right axis in fig. 2 . The increase of the ratio of output energies of the two pump pulses is seen to start at the input energy where the single pulse gain starts to saturate. This clearly indicates a strong patterning effect for this device when operated in the saturation regime where most signal processing schemes operate.

Also the recovery times of the last pump pulse are altered by the presence of the first pump pulse. This effect is illustrated in Fig. 3. The figure shows the characteristic times of the gain recovery after each pump pulse found by fitting the measured gain changes immediately following the pulse to a biexponential fitting function. The smallest of these times is denoted the relaxation time, $\tau_{R, 1}$ and $\tau_{R, 2}$.

The currents used in the measurements are all above the transparency current of $15 \mathrm{~mA}$.

The capture time of the first pump pulse, $\tau_{C, 1}$, is seen

to decrease slightly from $\approx 5 \mathrm{ps}$ at $20 \mathrm{~mA}$ bias to $3 \mathrm{ps}$ at 80 $\mathrm{mA}$. This decrease is attributed to an increase of the Auger assisted capture process at the higher currents where more carriers are present in both excited states of the dots and in the WL and barrier regions. For the second pulse however, the capture time is approximately $2 \mathrm{ps}$ higher for all currents. We attribute this increase to the removal of carriers in the WL by the first pulse, which again reduces the Auger assisted capture rate. The use of a double pump in the experiments thus indirectly reveals information about the carrier dynamics in the states above the active QD ground states.

The fact that the change in capture time for the two pump pulses does not change significantly when the current is increased indicates that for all currents shown here the amplification of a single strong pulse removes a significant amount of carriers from the WL. It could be expected that under full inversion of the WL the carrier depletion caused by the first pump would be insignificant compared with the large carrier density present in the WL. This would mean that no difference in capture time would be observed for the two pulses, i.e. no pattern effect would be present in this regard. It is however not possible to reach such high inversions in the device under investigation without introducing significant heating.

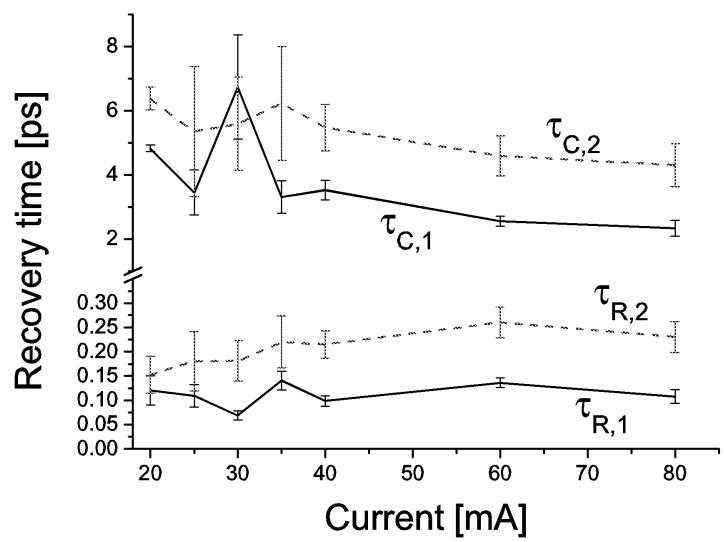

Fig. 3. Capture and intra dot relaxation time after the first and second pump pulses as function of bias current.

The extracted relaxation time, $\tau_{R, 1}$, is seen to be nearly constant at $\approx 0.13 \mathrm{ps}$, which corresponds to the width of the pump pulses. The majority of the instantaneous gain change is thus caused by two-photon absorption and this part of the gain change clearly recovers on the timescale of the pump pulse. For the second pulse however the fast recovery time is seen to be consistently longer and increases from $0.15 \mathrm{ps}$ at $20 \mathrm{~mA}$ to $\approx 0.25 \mathrm{ps}$ at $80 \mathrm{~mA}$. We ascribe this increase to enhanced participation of intra dot relaxation in the recovery process. Thus at $20 \mathrm{~mA}$ the device only exhibit a small modal gain and, therefore, only a minor fraction of the instantaneous gain change is a result of the removal of carriers. As the current is increased this fraction increases and since the first pump pulse removes a large part of the carriers in the excited states of each QD, the intradot relaxation time becomes increasingly important, which is reflected by the increase of the fast recovery time. It is important to note that even the fast recovery time is strongly dependent on the presence of the second pump pulse despite the fact that the pulses are separated by a significantly larger time. The carrier dynamics of the excited states and WL thus have a profound influence on the gain recovery of the QD ground state, which prohibits continuous fast gain recovery at ultra high bitrates.

In conclusion we have used pump-probe spectroscopy with a double pump pulse to investigate the potential for ultra high-speed signal processing in a QD device and find that the slower carrier dynamics of the excited states and WL causes significant patterning effects.

\section{Acknowledgement}

This work was supported by the EC via the project DOTCOM of the IST (project no. IST-2000-28713).

[1] T.W. Berg, J. Mørk, Appl. Phys. Lett., vol. 82, pp. 3083, 2003. [2] T. Akiyama et al., OFC Proceedings, paper PDP12, 2004

[3] T. Akiyama et al., Physica Status Solidi B, vol. 238, pp. 301304, 2003.

[4] P. Borri et al., IEEE J. Photon. Technol. Lett. 12(6), p. 594 (2000)

[5] T.W. Berg et al., IEEE J. Photon. Technol Lett. 13(6), p. 541, (2001)

[6] P. Borri et al., Opt. Comm., vol. 169, p. 317 (1999)

[7] A. Kovsh et al., J. Crystal Growth 250, 729 (2003) 